\title{
Protektive Beatmung reduziert postoperative pulmonale Komplikationen Contra
}

\author{
Martin Beiderlinden
}

Die „protektive Beatmung“ wurde bei Patienten mit ARDS etabliert. Ziel war es, die Entstehung von beatmungsassoziierten Lungenschäden (VILI) zu reduzieren, indem PEEP und Tidalvolumen $\left(V_{t}\right)$ individualisiert eingestellt wurden. Aktuell wird diese Form der Beatmungstherapie nicht mehr favorisiert und der Begriff der „protektiven Beatmung“ der Beatmung mit $\mathrm{V}_{\mathrm{t}} 6 \mathrm{ml} / \mathrm{kg}$ gleichgesetzt. Dabei kann auch dieses niedrige $V_{t}$ bei einigen Patienten mit ARDS VILI entstehen lassen. Obwohl bei beatmeten Lungengesunden die Gefahr der VILI-Entstehung erst ab $V_{t}$ von $>17 \mathrm{ml} / \mathbf{k g}$ besteht, wird in Analogie zur ARDS-Therapie eine intraoperative „protektive Beatmung“ intensiv untersucht.

Definition Unter protektiver Beatmung wird klassischerweise eine individuelle Beatmungsstrategie beim akuten Lungenversagen (ARDS) verstanden mit dem Ziel, VILI und die Freisetzung der proinflammatorischen Mediatoren (Biotrauma) durch Derekrutierung (Atelekttrauma) und Überdehnung (Baro-Volu-Trauma) zu verhindern [1].

\section{„Protektive“ Beatmung beim ARDS}

Individualisierte Beatmungstherapie Amato individualisierte die Beatmungstherapie bei Patienten mit ARDS [2]: Er begrenzte nach patienteneigenen Ruhedehnungskurven

- den PEEP 2 mbar oberhalb des unteren Umschlagpunkts und

- den Spitzendruck unter druckkontrollierter Beatmung 2 mbar unterhalb des oberen Umschlagpunkts.

16 mbar betrug der PEEP, um Derekrutierung zu verhindern, und ein Spitzendruck von $<30 \mathrm{mbar}$, was einem $V_{t}$ von etwa $6 \mathrm{ml} / \mathrm{kg}$ gleichkam, sollte Überdehnung vermeiden. Die Sterblichkeit war im Vergleich zu konventionell beatmeten Patienten signifikant reduziert (PEEP 7 mbar und $V_{t}$ $12 \mathrm{ml} / \mathrm{kg}$ ). Der positive Effekt dieser individuellen Beatmungseinstellung wurde durch Villar bestätigt, indem bereits nach der ersten Interimsanalyse eine signifikante Mortalitätsreduktion in der protektiven Gruppe zum Abbruch der Studie führte [3]. Diese „protektive Beatmung“ setzte sich in der ARDS-Therapie nicht durch.

Goldstandard Stattdessen ist das $\mathrm{V}_{\mathrm{t}}$ von $6 \mathrm{ml} / \mathrm{kg}$ bei Patienten mit akutem Lungenversagen [4] der "Goldstandard" und wird mittlerweile auch als Qualitätsindikator im Peer-Review-Verfahren der Deutschen interdisziplinären Vereinigung für Intensiv- und Notfallmedizin (DIVI) zur Qualitätssicherung in der Intensivmedizin geführt [5]. Dieser "Goldstandard“ hat mit der individualisierten, „protektiven“ Beatmungstherapie wenig gemeinsam, da alle Patienten ungeachtet der pulmonalen Pathophysiologie mit dem gleichen Atemzugvolumen beatmet werden. Der Derekrutierung (PEEP-Einstellung) wird nicht individuell, sondern anhand von $\mathrm{FiO}_{2} / \mathrm{PEEP}$-Tabellen begegnet $\left(\mathrm{FiO}_{2}=\right.$ inspiratorische $\mathrm{O}_{2}$-Konzentration). Dabei spielt die Schwere des Lungenversagens eine zentrale Rolle, denn bei Patienten mit nur mildem ARDS, gemessen an der statischen Compliance, konnte kein Unterschied zwischen einem $V_{t}$ von 6 oder $12 \mathrm{ml} / \mathrm{kg}$ nachgewiesen werden [4]. Diese Beobachtung lässt vermuten, dass erst eine näher zu definierende Lungenschädigung vorliegen muss, bevor eine Beatmung mit niedrigen Atemzugvolumina "protektiv“ quoad vitam ist.

Auch unter "protektiver Beatmung“ mit $6 \mathrm{ml} / \mathrm{kg}$ können regionale Überblähungen mit der Gefahr von VILI entstehen, die erst durch Reduktion auf $3 \mathrm{ml} / \mathrm{kg}$ unter Einsatz extrakorporaler Ventilation verhindert werden [6]. Nichtsdestotrotz wird unter „protektiver Beatmung“ nunmehr nur noch die Beatmung mit $6 \mathrm{ml} / \mathrm{kg}$ verstanden.

Der Begriff der „protektiven Beatmung“ stammt aus der Beatmungstherapie bei Patienten mit ARDS. Sie zeichnet sich aus durch eine Beatmung zwischen den individuell gewonnenen unteren und oberen Umschlagpunkten der Lungendehnungskurve eines Patienten. Dadurch können individuell der PEEP und - unter druckbegrenzter Beatmung - der Spitzendruck und somit das $V_{t}$ individuell adjustiert werden. 


\section{PEEP beim ARDS}

Wirkungsweise des PEEP PEEP soll - theoretisch - vor dem periodischen Öffnen und Schließen von rekrutierbaren Alveolen schützen und deshalb oberhalb des unteren Umschlagpunkts liegen, um Atelektrauma zu reduzieren. Allerdings konnten gleich 3 Studien keinen Überlebensvorteil bei Patienten mit ARDS durch eine Beatmung mit einem höheren gegenüber einem niedrigeren PEEP nachweisen [7-9]. Die standardisiert applizierten PEEP lagen in allen Studien unterhalb des von Amato individuell eingestellten von etwa 16 mbar. So wurden in den letztgenannten Studien Rescue-Therapien (NO, Bauchlage) in beiden Low-PEEP-Armen doppelt so häufig angewandt und die Sterblichkeit durch Hypoxämie (12 vs. $6,6 \%$ ) war auch knapp doppelt so hoch wie in den High-PEEP-Gruppen. Der PEEP in den High-PEEP-Gruppen war aber niedriger als unter individualisierter Einstellung [2, 3].

- Metaanalysen weisen darauf hin, dass ein höherer PEEP im schweren ARDS vorteilhaft ist $[10,11]$, was auch Untersuchungen mit individuell titriertem PEEP zeigen konnten [12, 2].

Zukunft der Beatmung bei ARDS Dabei ist das ARDS keine uniforme Erkrankung, sondern ein Oberbegriff für akute pulmonale Hypoxämien. Bei gleicher Oxygenierungsbeeinträchtigung haben unterschiedliche Ätiologien unterschiedliche Bildmorphologien zur Folge ( $\bullet$ Abb. 1 und 2). Selbst eine „protektive“ Beatmung mit $\mathrm{V}_{\mathrm{t}} 6 \mathrm{ml} / \mathrm{kg}$ kann „injurious“ sein [6]. Liegt also die Zukunft der mechanischen Beatmung des ARDS-Patienten in noch niedrigeren $V_{t}[13]$ oder gar in Gattinonis „lung at rest“ mit extrakorporaler Ventilation und/oder Oxygenierung [14]?

Die „protektive Beatmung“ beim ARDS wird nicht angewandt. Denn anstatt einer individualisierten PEEP-Adjustierung und Ventilation mit individualisierten $\mathrm{V}_{\mathrm{t}}$ wird ein Standard- $\mathrm{V}_{\mathrm{t}}$ von $6 \mathrm{ml} / \mathrm{kg}$ als protektiv betrachtet und den DerekrutierungsKomponenten nur wenig Bedeutung beigemessen.

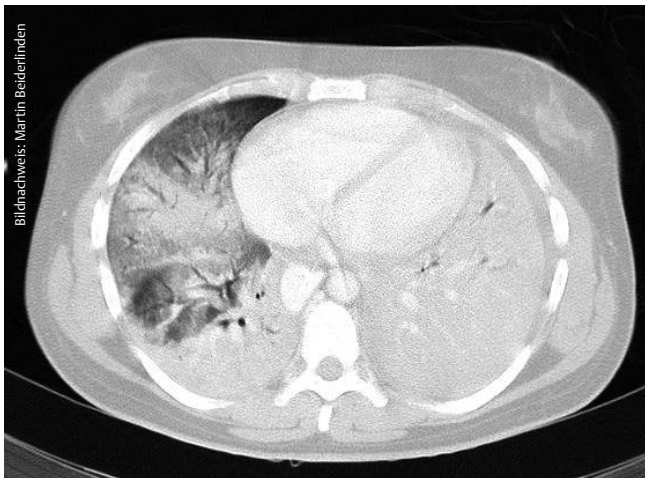

Neue Studienergebnisse Eine aktuelle retrospektive Untersuchung von Amato [15] stellt nun auch den protektiven Effekt der Ventilation mit einem $V_{t}$ von $6 \mathrm{ml} / \mathrm{kg}$ selbst beim ARDS in Frage. Aus den gepoolten Daten der ARDS-NetworkStudien konnte eine Senkung der Sterblichkeit durch ein $V_{t}$ von $6 \mathrm{ml} / \mathrm{kg}$ nur bei den Patienten beobachtet werden, bei denen der "driving pressure“ (also die Differenz aus Plateaudruck und PEEP) $<15$ mbar betrug. Nun steht die Hypothese im Raum, dass nicht das niedrige Tidalvolumen protektiv ist, sondern der dafür erforderliche „driving pressure“. Dieser wiederum hängt von der Compliance der Lunge und Thoraxwand ab. Weitere prospektive Studien werden folgen müssen, um den Effekt dieses Konzepts zu evaluieren.

- Nichtsdestotrotz ist das einmal mehr ein Indiz, dass eine generelle $\mathrm{V}_{\mathrm{t}}$-Reduktion der komplexen Pathophysiologie des ARDS nicht gerecht wird und eine individualisierte Herangehensweise analog des Konzepts der „klassischen“ protektiven Beatmung neu zu bewerten ist.

\section{"Protektive Beatmung“ bei Lungengesunden}

Übertragung der Beatmungsstrategien Trotz dieser terminologischen und methodischen Unsicherheiten in der Beatmungstherapie bei ARDSPatienten soll nun der Terminus ,protektive Beatmung" auf Lungengesunde übertragen werden. - Abb. 3 zeigt exemplarisch das Thorax-CT eines beatmeten Lungengesunden. Nicht nur von der Bildmorphologie fällt es schwer zu verstehen, warum für Lungengesunde mit vielfach kürzerer (intraoperativer) Beatmungszeit verglichen mit ADRS-Patienten die gleichen Beatmungsstrategien zur Anwendung kommen sollen. Während Beatmung mit $V_{t}$ von $6 \mathrm{ml} / \mathrm{kg}$ bei ARDS-Patienten bereits zur Entstehung von VILI beitragen kann, so scheint bei beatmeten Lungengesunden diese Gefahr der VILI-Entstehung erst ab $V_{t}$ von $17-22 \mathrm{ml} / \mathrm{kg}$ gegeben zu sein [16].

Physiologisches $V_{t}$ Wie hoch ist eigentlich das physiologische $V_{t}$ ? Spirometrische Daten suggerieren, dass unter Ruhebedingungen das „physio-

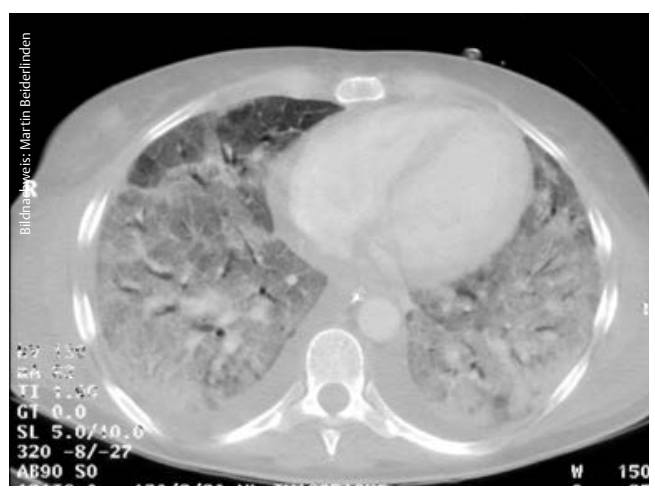

Abb. 1 (links) Thorax-CT eines Patienten mit schwerem ARDS auf dem Boden einer ambulant erworbenen Pneumonie (Pneumokokken). Atemstillstand PEEP 10 mbar während der Untersuchung, P/F-Quotient $80 \mathrm{mmHg}$. Es zeigen sich vornehmlich ausgeprägte Konsolidierungen der linken Lunge und des Unterlappens rechts. Milchglasartige Trübungen („ground glass opacities") im Mittellappen.

Abb. 2 (rechts) Thorax-CT eines Patienten mit schwerem ARDS auf dem Boden einer Peritonitis nach Hohlorganperforation. Atemstillstand PEEP 10 mbar während der Untersuchung, P/F-Quotient $100 \mathrm{mmHg}$. Angedeutete „klassische Dreiteilung" der rechten Lunge. Überwiegend milchglasartige Trübungen ("ground glass opacities") mit angedeuteten basalen Konsolidierungen. 
Abb. 3 Thorax-CT eines Patienten mit isoliertem SchädelHirn-Trauma 20 min nach problemloser Intubation unter PEEP 5 mbar. Minimale basale Minderbelüftungen (Atelektasen).

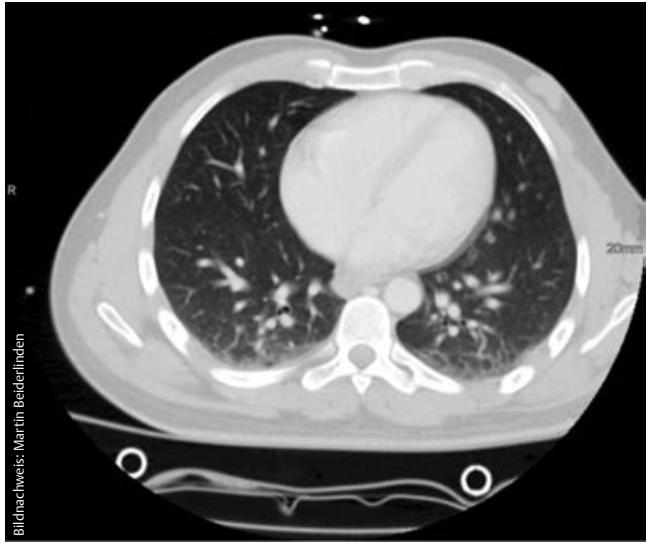

logische“ $V_{t} 6-8 \mathrm{ml} / \mathrm{kg}$ beträgt. Spirometrische Untersuchungen sind aber keine physiologischen Bedingungen, denn sie betrachten nur einen kurzen Zeitraum. Bendixen untersuchte junge Erwachsene unter Ruhebedingungen über einen Zeitraum von $>1 \mathrm{~h}$. Das durchschnittliche $V_{t}$ betrug zwar $6-8 \mathrm{ml} / \mathrm{kg}$, zeigte aber eine ausgeprägten Atemzug-zu-Atemzug-Variabilität. Darüber hinaus machten die Probanden alle 6 min einen „Seufzer“, der definiert war als mind. das 3-Fache des durchschnittlichen $V_{t}$. Bei Frauen betrug das Seufzervolumen 1,2l und bei Männern 1,8l, etwa $50 \%$ der Vitalkapazität. Unter physiologischen Bedingungen vollzogen also Frauen und Männer alle $6 \mathrm{~min}$ ein $V_{\mathrm{t}}$ von 20 bzw. $24 \mathrm{ml} / \mathrm{kg}$ [17]. Von ambitionierten Radsportlern ist bekannt, dass sie bei Ausbelastung ein Atemminutenvolumen von $1641 / \mathrm{min}$ mit einem $V_{t}$ von $40 \mathrm{ml} / \mathrm{kg}$ und bei mehrstündigen Radrennen etwa $35 \mathrm{ml} / \mathrm{kg}$ zeigen [18]. Aus tierexperimentellen Daten wissen wir, dass die Schädigung einer Lunge durch hohes Atemzugvolumen unabhängig davon ist, ob das Volumen mit Überdruck oder Unterdruck verabreicht wurde [18].

\footnotetext{
Unter physiologischen Bedingungen beträgt das $V_{\mathrm{t}} 6-8 \mathrm{ml} / \mathrm{kg}$, variiert aber stark mit tiefen Atemzügen alle $6 \mathrm{~min}$ mit einem $V_{\mathrm{t}}$ von $20-24 \mathrm{ml} / \mathrm{kg}$. $V_{t}$ können bei Ausdauersportlern über einen langen Zeitraum Werte bis zu $40 \mathrm{ml} / \mathrm{kg}$ erreichen. Bei Gesunden ist das $V_{t}$ auch unter physiologischen Bedingungen durchaus „unprotektiv“.
}

Umfrage zur intraoperativen Beatmung Einer multizentrischen internationalen Umfrage zur intraoperativen Beatmungstherapie zufolge beträgt das $V_{t} 6-8 \mathrm{ml} / \mathrm{kg}$. In $>80 \%$ wird ein PEEP von mind. 5 mbar appliziert. 16\% der Befragten führen routinemäßig Rekrutierungsmanöver durch [20]. Interessanterweise hat sich an der Klinik für Anästhesiologie der Universitätsklinik Düsseldorf das $\mathrm{V}_{\mathrm{t}}$ stetig von knapp $15 \mathrm{ml} / \mathrm{kg}$ (1994) auf $<7 \mathrm{ml} / \mathrm{kg}$ (2009) verringert, bei konstantem PEEP von $5 \mathrm{mbar}$ (Treschan, persönliche Kommunikation).
Diese Ergebnisse zeigen, dass die in der Intensivmedizin applizierten niedrigen $\mathrm{V}_{\mathrm{t}}$ schleichend Einzug in den OP genommen haben, ohne jedoch hierfür validiert worden zu sein.

Metaanalyse zur protektiven Beatmung Eine Metaanalyse zeigt die Überlegenheit einer „protektiven Beatmung“ im Hinblick auf die Entstehung von akutem Lungenversagen (ALI), Pneumonie und Mortalität. Bei dieser Untersuchung wurden beatmete Nicht-ARDS-Patienten und intraoperative Patienten ausgewertet. Allein der durchschnittliche P/F-Quotient von knapp $>300 \mathrm{mmHg}$ lässt an der Übertragbarkeit der Ergebnisse auf intraoperative Beatmungsstrategien für Lungengesunde zweifeln [21].

Studie zu postoperativen pulmonalen Komplikationen Eine multizentrische Studie aus Frankreich untersuchte den Einfluss einer „protektiven Beatmung“ bei abdominalchirurgischen Eingriffen im Hinblick auf das Auftreten postoperativer pulmonaler Komplikationen. In der Gruppe der Patienten, die „protektiv“ beatmet wurden, traten diese hochsignifikant seltener auf, ohne die Sterblichkeit positiv zu beeinflussen. Trotz der Fallzahl $(n=400)$ und der hochrangigen Publikation als Maß für die Qualität der Studie sind der Informationsgewinn und die Übertragbarkeit in die klinische Praxis leider sehr begrenzt [22]. In der "protektiven“ Gruppe wurden gleich 3 Beatmungsparameter im Vergleich zur Kontrollgruppe verändert: PEEP (6-8 vs. 0 mbar), $\mathrm{V}_{\mathrm{t}}$ ( 6 vs. $12 \mathrm{ml} / \mathrm{kg}$ ) und Rekrutierungsmanöver (alle $30 \mathrm{~min}$ wurde für $30 \mathrm{~s}$ ein PEEP von 30 mbar appliziert vs. keine Rekrutierungsmanöver).

- Ob dieses „protektive“ Beatmungsregime überhaupt für die Senkung der Inzidenz pulmonaler Komplikationen verantwortlich gemacht werden darf, kann bezweifelt werden. In der Kontrollgruppe gab es fast doppelt so viele Anastomoseninsuffizienzen wie in der "protektiven“ Gruppe (44 vs. $24, \mathrm{p}=0,009$ ).

Es ist daher nicht unwahrscheinlich, dass der beobachtete protektive Effekt eher der geringeren Komplikationsrate anzurechnen ist.

PROVHILO-Studie Inwieweit sich ein höherer PEEP auf die Derekrutierungskomponente und somit auf die postoperativen pulmonalen Komplikationen auswirkt, wurde in der PROVHILOStudie untersucht. 900 abdominalchirurgische Patienten wurden multizentrisch randomisiert: Ein PEEP von 12 mbar und Rekrutierungsmanöver (intraoperativ und vor Extubation) oder ein PEEP $<2$ mbar ohne Rekrutierungsmanöver. Patienten beider Gruppen wurden mit einem $V_{t}$ von $<8 \mathrm{ml} /$ $\mathrm{kg}$ beatmet [23]. 
- Erwartungsgemäß verursachte der um $10 \mathrm{mbar}$ höhere intrapulmonale Druck in der Behandlungsgruppe signifikant mehr hämodynamische Instabilitäten - mit der Konsequenz der höheren kristallinen Flüssigkeitszufuhr und dem vermehrten Einsatz vasoaktiver Substanzen. Die postoperative pulmonale Komplikationsrate wurde jedoch nicht beeinflusst.

Es sei hier angemerkt, dass in der „High-PEEP“Gruppe Lungengesunden ein PEEP von $12 \mathrm{mbar}$ verabreicht wurde, der höher war als in der Kontrollgruppe bei ARDS-Patienten [7-9]. Die Schlussfolgerung der Autoren, dass eine Beatmungsstrategie für diese Patientenklientel aus niedrigem PEEP ohne Rekrutierungsmanöver und niedrigem $V_{t}$ bestehen sollte, überrascht insofern, als die Höhe des $V_{t}$ nicht Bestandteil der Untersuchung war.

Übliche Beatmungsstrategie in Deutschland Treschan untersuchte eine intraoperative Beatmungsstrategie, die vielleicht der Praxis in Deutschland am nächsten kommt: 100 Patienten zu großen Oberbaucheingriffen wurden randomisiert mit einem $V_{t}$ entweder von 6 oder $12 \mathrm{ml} /$ $\mathrm{kg}$ beatmet. Alle Patienten erhielten einen PEEP von 5 mbar und Rekrutierungsmanöver vor Extubation. Unter dieser Beatmung kam es in der Gruppe mit $V_{t} 6 \mathrm{ml} / \mathrm{kg}$ zu einem signifikant niedrigeren arteriellen $\mathrm{O}_{2}$-Partialdruck $\left(\mathrm{paO}_{2}\right)$ unter Allgemeinanästhesie, der auch noch am 5. postoperativen Tag unterschiedlich blieb (69 vs. $78 \mathrm{mmHg}$ ). In der $V_{\mathrm{t}} 6 \mathrm{ml} / \mathrm{kg}$ Gruppe konnten bei $88 \%$ der Patienten, in der $V_{t} 12 \mathrm{ml} / \mathrm{kg}$ Gruppe nur bei $68 \%$ radiologisch Atelektasen nachgewiesen werden - ein signifikanter Unterschied. Trotz eines PEEP von $5 \mathrm{mbar}$ und Rekrutierungsmanövern konnte die Ausbildung von Atelektasen nicht befriedigend verhindert werden. Niedrige $V_{t}$ scheinen aber die Ausbildung von Atelektasen eher zu verstärken [24].

Höhere Sterblichkeit bei $V_{t} 5-8 \mathrm{ml} / \mathrm{kg}$ Eine retrospektive Analyse zum Einfluss des $V_{t}$ unter einem PEEP von 4 mbar bei $>29000$ Patienten zeigt, dass Beatmung mit einem $V_{t} 5-8 \mathrm{ml} / \mathrm{kg}$ mit einer signifikant höheren Sterblichkeit assoziiert ist als eine mit $8-10 \mathrm{ml} / \mathrm{kg}$ und $10-12 \mathrm{ml} / \mathrm{kg}$ [25].

Was genau ist lungenprotektive Beatmung? Auch wenn die aktuellste, sehr aufwendige Metaanalyse eine Senkung der Inzidenz der postoperativen pulmonalen Komplikation bei abdominalund thoraxchirurgischen Patienten durch „lungenprotektive“ Beatmungsstrategien dokumentiert, so bleibt die Frage unbeantwortet, was unter „lungenprotektiv“ zu verstehen ist. Denn in diese Meta-Analyse mündeten die oben aufgeführten Untersuchungen mit den unterschiedlichen Beatmungsstrategien und beschriebenen methodischen Problemen [26]. Daher ist es verfrüht, Beatmungsstrategien mit niedrigen $\mathrm{V}_{\mathrm{t}} \mathrm{zu}$ favori- sieren, solange nicht geklärt ist, für welche Patientenklientel sie einen Vorteil generieren.

- Die aktuelle Literatur rechtfertigt nicht den Schluss, dass mit protektiver Beatmung, was auch immer darunter zu verstehen sein mag, postoperative pulmonale Komplikationen zu reduzieren seien.

Im Gegenteil: Beatmung mit niedrigen $V_{t}$ kann mit Beeinträchtigung der Oxygenierung bis hin zu einer erhöhten Mortalität vergesellschaftet sein.

Heutiger Stand „Protektive Beatmung“ mit seiner individuellen PEEP- und $\mathrm{V}_{\mathrm{t}}$-Einstellung wird selbst bei ARDS-Patienten, für die sie konzipiert wurde, nicht allumfänglich praktiziert. Stattdessen hat sich die Ventilation mit $V_{t} 6 \mathrm{ml} / \mathrm{kg}$ als „pars pro toto" unter diesem Begriff durchgesetzt und der Derekrutierungs-Komponente wird weniger Bedeutung beigemessen. Neuere Daten stellen sogar den benefiziellen Effekt der Ventilation mit $V_{t} 6 \mathrm{ml} / \mathrm{kg}$ infrage [15]. Diese Strategie auf Lungengesunde zu übertragen, erscheint aus vielerlei Aspekten unbegründet, wenn nicht gefährlich: Die Höhe des PEEP ist für Lungengesunde und auch für Risikopatienten (pulmonale Vorerkrankungen, Oberbauch- und Thoraxeingriffe) unklar. Niedrige $V_{t}$ sind mit Oxygenierungsstörungen aufgrund Atelektasenbildung und mit einer erhöhten perioperativen Sterblichkeit assoziiert.

Fazit Es werden Studien folgen müssen, die den idealen PEEP und das ideale $V_{t}$ in Abhängigkeit vom Risikoprofil des Patienten und des operativen Eingriffs beschreiben. Eine generelle Standardbeatmung wird diesem komplexen Sachverhalt nicht gerecht. Die derzeitige Datenlage zurzeit rechtfertigt nicht die These, dass „protektive“ Beatmung pulmonale Komplikationen reduziert, nicht nur wegen der unzureichenden Definition von „protektiv“. 4

Kernaussagen

Der Begriff der „protektiven Beatmung“ stammt aus der Beatmungstherapie bei Patienten mit ARDS. Sie zeichnet sich aus durch eine Beatmung zwischen den individuell gewonnenen unteren und oberen Umschlagpunkten der Lungendehnungskurve eines Patienten.

Die „protektive Beatmung“ beim ARDS wird nicht angewandt. Denn anstatt einer individualisierten PEEP-Adjustierung und Ventilation mit individualisierten $\mathrm{V}_{\mathrm{t}}$ wird ein Standard- $V_{t}$ von $6 \mathrm{ml} / \mathrm{kg}$ als protektiv betrachtet und den DerekrutierungsKomponenten nur wenig Bedeutung beigemessen.

Die in der Intensivmedizin applizierten, niedrigen $\mathrm{V}_{\mathrm{t}}$ haben schleichend Einzug in den OP genommen, ohne jedoch hierfür validiert worden zu sein. 


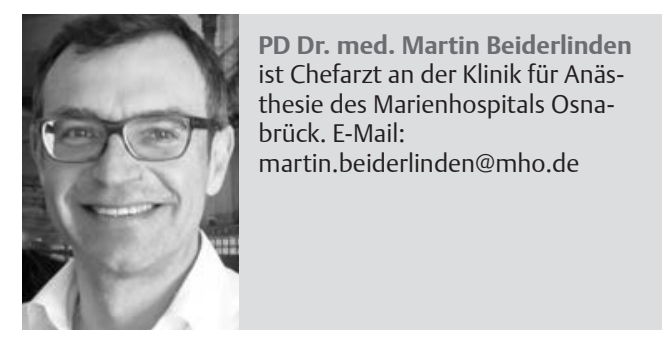

Literaturverzeichnis

1 Tusman G, Böhm SH, Warner DO, Sprung J. Atelectasis and perioperative pulmonary complications in high risk patients. Curr Opin Anesthesiol 2012; 25: 1-10

2 Amato MB, Barbas CS, Medeiros DM et al. Effect of a protective-ventilation strategy on mortality in the acute respiratory distress syndrome. N Engl J Med 1998; 338: 347354

3 Villar, J, Kacmarek RM, Pérez-Méndez L, Aguirre-Jaime A for the ARIES Network. A high positive end-expiratory pressure, low tidal volume ventilatory strategy improves outcome in persistent acute respiratory distress syndrome: $A$ randomized, controlled trial. Crit Care Med 2006; 34: 13111318

4 ARDS Network. Ventilation with lower tidal volumes as compared with traditional tidal volumes for acute lung injury and the acute respiratory distress syndrome. $N$ Engl J Med 2000; 342: 1301-1308

5 DIVI. Hauptindikatoren 1-10. Im Internet: www.divi.de/qualitaetssicherung/peer-review/ qualit\%C3\%A4tsindikatoren.html

6 Terraggni PP, Rosboch G, Tealdi A et al. Tidal hyperinflation during low tidal volume ventilation in acute respiratory distress syndrome. Am J Respir Crit Care Med 2007; 175: 160-166

7 The National Heart, Lung, and Blood Institute ARDS Clinical Trials Network. Higher versus lower positive end-expiratory pressures in patients with the acute respiratory distress syndrome. N Engl J Med 2004; 351: 327-336

8 Mercat A, Richard JCM, Vielle B et al., for the Expiratory Pressure (Express) Study Group. Positive end-expiratory pressure setting in adults with aute lung injury and acute respiratory distress syndrome. JAMA 2008; 299: 646-655

9 Maede MO, Cook DJ, Guyatt GH et al., for the Lung Open Ventilation Study Investigators. Ventilation strategy using low tidal volumes, recruitment maneuvers, and high positive end-expiratory pressure for acute lung injury and acute respiratory distress syndrome. JAMA 2008; 299: 637-645

10 Phoenix SI, Paravastu S, Columb M et al. Does a higher expiratory pressure decreas mortality in acute respiratory distress syndrome? A systematic review and meta-analysis. Anesthesiology 2009; 110: 1098-1105

11 Briel M, Maede M, Mercat A et al. Higher versus lower positive end-expiratory pressure in patients with acute lung injury and acute respiratory distress syndrome: Systemic review and meta-analysis. JAMA 2010; 303: 865-873

12 Talmor D, Sarge T, Malhotra A et al. Mechanical ventilation guided by esophageal pressure in acute lung injury. N Engl J Med 2008; 359: 2095-2104

13 Bein T, Weber-Carstens S, Goldmann A et al. Lower tidal volume strategy $(3 \mathrm{ml} / \mathrm{kg}$ ) combined with extracorporal $\mathrm{CO}_{2}$ removal versus "conventional " protective ventilation $(6 \mathrm{ml} / \mathrm{kg})$ in severe ARDS: the prospective randomized Xtravent-study. Intensive Care Med 2013; 39: 847-856
Interessenkonflikt Der Autor erklärt, dass keine Interessenkonflikte vorliegen.

Beitrag online zu finden unter http://dx.doi. org/10.1055/s-0041-105804

14 Gattinoni L, Pesenti A, Marcolin R et al. Low-frequency positive-pressure ventilation with extracorporeal $\mathrm{CO}_{2}$ removal in severe acute respiratory failure. JAMA 1986; 256: 881-886

15 Amato MBP, Meade MO, Slutsky AS et al. Driving pressure and survival in the acure respiratory distress syndrome. $N$ Engl J Med 2015; 372: 747-755

16 Gattinoni L, Protti A, Caironi P, Carlesso E. Ventilator-induced lung injury: the anatomical and physiological framework. Crit Care Med 2010; 38: 10 [Suppl]

17 Bendixen HH, Smith GM, Mead J. Pattern of ventilation in young adults. J Appl Physiol 1964; 19: 195-198

18 Turner LA, Tecklenburg-Lund SL, Chapman RF et al. Inspiratory muscle training lowers the oxygen cost of voluntary hyperpnea. J Appl Physiol 2012; 112: 127-134

19 Dreyfuss $D$, Basset G, Soler P, Saumon G. High inflation pressure pulmonary edema: respective effects of high airway pressure, high tidal volume, and positive end-expiratory pressure. Am Rev Respir Dis 1988; 137: 1159-1164

20 Treschan T, Werth A, Hemmes SNT et al. How do anesthesiologist apply intra-operative ventilation? The international survey on ventilation practice (iVent): 5AP3-2. Eur J Anesthesiol 2014; 31: 82

21 Neto AS, Cardoso SO, Manetta JA et al. Association between use of lung-protective ventilation with lower tidal volumes and clinical outcomes among patients without respiratory distress syndrome: A meta-analysis. JAMA 2012: 308: 1651-1659

22 Futier E, Constantin JM, Paugam-Burtz C et al., for the IMPROVE Study Group. A trial of intraoperative low-tidalventilation in abdominal surgery. N Engl J Med 2013; 369: 428-437

23 The PROVE Network Investigators. High versus low positive end-expiratory pressure during general anaesthesia for open abdominal surgery (PROVHILO trial): a multicentre randomised control trial. Lancet 2014; 384; 495-503

24 Treschan TA, Kaisers W, Schaefer MS et al. Ventilation with low tidal volumes during upper abdominal surgery does not improve postoperative lung function. $\mathrm{Br} J$ Anaesth 2012; 109: 263-271

25 Levin MA, McCormick PJ, Lin HM et al. Low intraoperative tidal volume ventilation with minimal PEEP is associated with increased mortality. Br J Anaesth 2014; 113: 97-108

26 Neto AS, Hemmes SNT, Barbas CSV et al. Incidence of mortality and morbidity related to postoperative lung injury in patients who have undergone abdominal or thoracis surgery: a systemic review and meta-analysis. Lancet Respir Med 2014; 2: 1007-1015 